\title{
Reflexividad y prácticas de investigación entre académicos de la comunicación en México
}

La investigación sobre los fenómenos de comunicación en América Latina preserva su actitud crítica en la formulación de hipótesis teóricas y en la delimitación de premisas analíticas. Sin embargo, intensifica el uso de procedimientos empíricos (cuantitativos y cualitativos) para describir y diagnosticar situaciones. Esta postura corresponde a un sentimiento consensual de que la investigación científica representa un instrumento vital para construir sociedades democráticas prósperas y pluralistas, una utopía que moviliza a los investigadores de la comunicación ante el umbral del siglo XXI.

Marques de Melo, 1994: 428-429.

\begin{abstract}
A
PARTIR DE UN ESTUDIO multidimensional realizado acerca de los factores socioculturales determinantes de la estructuración del campo de la investigación académica de la comunicación en México (Fuentes, 1995), en este trabajo se exponen sintéticamente los planteamientos metodológicos y los resultados empíricos obtenidos en referencia a la configuración cognoscitiva del campo por parte de un grupo de investigadores que, como sujetos concretamente situados, operan en la práctica cotidiana, desde las instituciones en que trabajan, un proyecto profesional, científico y social que al mismo tiempo que subyace en su estilo de pensamiento, define (ante ellos mismos y ante otros) su identidad específica.

La configuración cognoscitiva del campo de la investigación académica de la comunicación en México se abordó heurísticamente en el estudio citado mediante el análisis de tres niveles: el propiamente científico, el profesional y el ideoló-

1. Instituto Tecnológico y de Estudios Superiores de Occidente (ITESO), Departamento de Estudios Socioculturales. Universidad de Guadalajara (UdeG), Departamento de Estudios de la Comunicación Social.
\end{abstract}


gico. En el primero de estos niveles se exploraron las convergencias y divergencias conceptuales y metodológicas detectadas bibliométricamente en los productos publicados de la investigación, y sus resultados no se presentan aquí (véanse Fuentes, 1996a, 1996b).

En el segundo nivel se buscaron los elementos comunes y los diversos de los estilos de pensamiento prevalecientes entre los investigadores, y en el tercero, la instrumentalización del conocimiento producido en función tanto de la constitución de una identidad colectiva ante el exterior del campo como de una jerarquización interna de los investigadores en el campo de la producción, reproducción y aplicación de ese conocimiento. En estos dos últimos niveles se trabajó, mediante dos acercamientos distintos, sobre las representaciones de los sujetos acerca de su propia práctica, en términos de reflexividad. En estas páginas se exponen los resultados correspondientes al análisis del segundo nivel.

Teóricamente, el supuesto es que las características cognoscitivas de un campo multidisciplinario de investigación, como el de la comunicación en México, exigen la ampliación del rango de "objetos del compromiso de grupo" que Thomas S. Kuhn llamaba paradigma o matriz disciplinaria (Kuhn, 1982: 321) para explicar la relación entre ese "conjunto de hábitos (intelectuales, verbales, conductuales, mecánicos, tecnológicos...)" o "paradigma sociológico", como distinguió M. Masterman (1970: 66), y una comunidad científica. Esto significa reconstruir los elementos constitutivos del estilo de pensamiento de ese colectivo (Fleck, 1979: 39), enfatizando las configuraciones específicas (ideológicas) de las representaciones o imágenes mentales compartidas sobre los problemas de interés, el juicio que el grupo considera evidente, y los métodos aplicados como "medios de cognición" (Fleck, 1979: 99).

El concepto de estilo de pensamiento de L. Fleck se asemeja sorprendentemente al habitus de P. Bourdieu (1988a; 1988b) y al esquema interpretativo de A. Giddens (1984). Sin entrar aquí en detalles sobre las tramas conceptuales subyacentes, se utiliza para sustentar teóricamente, por un lado, el carácter dialéctico de la mutua constitución entre prácticas e identidades subjetivas y los campos o sistemas objetivos, y por otro, la posibilidad de objetivar esas relaciones mediante el análisis de las formaciones discursivas con que los sujetos se las representan como significados (que son el producto de la interpretación subjetiva de la información del entorno) y las comparten como sentido (que es el producto pragmático de la interacción comunicativa entre sujetos).

\section{Las proyecciones del estilo de pensamiento}

Un paso indispensable para analizar la conformación del habitus de "comunicólogo" es partir de una exploración de las creencias del grupo de sujetos de este estudio sobre algunas dimensiones de su propia práctica, exploración basada en la idea de que "las principales entidades sociales tienen siempre un aspecto simbólico, una cultura, así como una estructura social, ciertos relatos y creencias 
compartidas que coadyuvan a que los participantes definan quiénes son, qué hacen, por qué lo hacen, y si ello les implica un' infortunio o una bendición" (Clark, 1992: 113).

Mediante una técnica proyectiva (retomada de las escalas Likert, para la medición de actitudes) se buscó identificar algunos elementos de un perfil ideológico o formación discursiva, analizables estadísticamente, para comenzar a bosquejar el contenido y los grados de consenso que pudieran conformar el "estilo de pensamiento" compartido, interpretable como constitutivo de una matriz disciplinaria del campo.

Los datos provienen de una encuesta realizada entre octubre de 1992 y julio de 1993 (respondida por 41 investigadores, véase Anexo), y específicamente de la sección del cuestionario que presentaba a los sujetos 40 afirmaciones extraídas textualmente de la documentación publicada por investigadores mexicanos y latinoamericanos, referentes a algunas dimensiones, características y tendencias del propio campo. De cada una de las 40 citas se solicitó a los sujetos, primero, una apreciación de su acuerdo/desacuerdo en una escala de cinco grados, y, segundo, un comentario libre.

El supuesto metodológico es que los sujetos identificarían su postura ante ciertas interpretaciones (que presumiblemente les serían cercanas y, por tanto, relativamente reconocibles) mediante la expresión de su acuerdo/desacuerdo, pero sobre todo por los comentarios que las afirmaciones les suscitaran. Más que la medición de los grados de consenso particular que cada afirmación alcanzara, se trataba de detectar, por una parte, dimensiones polémicas de las representaciones explícitamente "públicas" de aspectos del campo y, por otra, mediante los comentarios, indicaciones del sentido subjetivo de los acuerdos/desacuerdos, es decir, de las operaciones de la lectura como producción de sentido, del "discurso en reconocimiento", siguiendo a Eliseo Verón:

La "lectura" (es decir, el "efecto de sentido") siendo necesariamente el punto de acceso al análisis de las operaciones discursivas, se encuentra frente a dos vías diferentes, que conducen a dos modelos: un modelo de la producción del discurso y un modelo del consumo del discurso. Estos dos modelos jamás coinciden exactamente. En otras palabras: en relación con un conjunto textual dado, y para un nivel determinado de pertinencia, siempre existen dos lecturas posibles: la del proceso de producción (de generación) del discurso y la del consumo, de la recepción de ese mismo discurso. Tomando prestada una fórmula de la lingüística, podemos decir que el funcionamiento de todo discurso depende no de una, sino de dos tipos de "gramáticas": de producción y de reconocimiento. Estos dos tipos de gramáticas jamás son idénticos (Verón, 1987: 20).

Debido a que los textos citados fueron producidos y consumidos (escritos y leídos) por el mismo tipo de sujetos (a veces los mismos individuos), se forzó así una confrontación de las "gramáticas" de producción y reconocimiento discursivos operadas por los sujetos en torno a referentes constitutivos de su propio campo especializado de conocimiento, de manera que se pudieran manifestar 
algunas de las convergencias y divergencias de las representaciones del campo por los sujetos que lo constituyen, desde el nivel de la terminología hasta el de las categorías de pensamiento, estructuras de significación y normas éticas (valoración de los objetos de referencia del discurso, mediante ciertos principios). Como exploración de la cultura propia del campo, no obstante; la aplicación de este instrumento no puede pretender más que la generación de indicios, tanto por cuestiones técnicas de validación estadística como, sobre todo, por la naturaleza disciplinaria del sistema de creencias objeto de análisis:

A mayor profesionalización de la ocupación, mayor separación cultural [en el campo universitario]. Por tanto, las escuelas de medicina y derecho parecen ostentar las culturas más peculiares que acumulan en el tiempo un conjunto de normas características de la ocupación [...] Las escuelas de las semiprofesiones en general, especialmente las que se basan en las ciencias sociales (por ejemplo, la educación y el trabajo social [o la comunicación]), muestran menor firmeza y claridad en la integración de sus costumbres. Esta tendencia tiene su raíz en la 'incertidumbre cognitiva'. En tanto mescolanzas de conocimientos extraídos de disciplinas tan inciertas como la psicología y la sociología, estos campos tienden a construir armazones simbólicos más difusos (Clark, 1992: 123).

Sin embargo, pueden comenzar a quedar en evidencia ciertos rasgos de la cultura de la especialidad, perteneciente específicamente a la comunidad de los investigadores mexicanos de la comunicación, operantes como "categorías de pensamiento esotéricas que [la] distinguen de las demás [comunidades disciplinarias]" (Clark, 1992: 119), es decir, como ingredientes de la configuración cognoscitiva que son constitutivos de la identidad propia del campo. De esta manera, los resultados obtenidos se utilizaron como insumos de un análisis de alcance mayor (véase Fuentes, 1997), y ni en el momento de ese análisis (entre uno y dos años después de la aplicación de la encuesta) ni en el momento de la actual publicación tienen la pretensión de ser conclusivos, y ni siquiera descriptivos de las representaciones actuales de los investigadores sobre el campo. Por ello, en términos estructurales ("estilos de pensamiento"), aunque no necesariamente en cada una de sus partes y menos en cuanto a sus referentes, se puede sostener como vigente el análisis y sus resultados.

Aunque en el cuestionario las 40 afirmaciones se presentaron a los sujetos en otro orden, se seleccionaron como hipotéticamente representativas de tres dimensiones del campo académico de la comunicación: la "práctica de la investigación", "la formación de investigadores" y "la profesión". En el cuadro 1 se presentan las medias y las desviaciones estándar de las calificaciones con que los sujetos definieron su acuerdo/desacuerdo con cada una de las afirmaciones que se les presentaron, sin referencia al autor, dentro de una escala de cinco grados $(-2$ a 2$)$. 


\section{CUADRO 1}

Afirmaciones sobre la investigación de la comunicación, calificadas por investigadores mexicanos

\begin{tabular}{|c|c|c|c|c|c|c|c|c|}
\hline $\begin{array}{c}\text { Sobre la } \\
\text { práctica de } \\
\text { la investiga- } \\
\text { ción }\end{array}$ & media & & $\begin{array}{c}\text { Sobre la } \\
\text { formación } \\
\text { de investiga- } \\
\text { dores }\end{array}$ & media & d.s. & $\begin{array}{c}\text { Sobre la } \\
\text { investigación } \\
\text { como } \\
\text { profesion } \\
\end{array}$ & media & d.s \\
\hline 01 & 1.325 & 0.888 & 03 & 1.300 & 1.090 & 02 & 1.525 & 0.933 \\
\hline 05 & 1.125 & 1.017 & 11 & 1.125 & 1.180 & 04 & 0.175 & 1.337 \\
\hline 07 & 0.600 & 1.410 & 13 & 1.100 & 1.057 & 06 & 1.050 & 1.339 \\
\hline 08 & -0.450 & 1.395 & 23 & 1.350 & 0.802 & 16 & 0.250 & 1.581 \\
\hline 09 & 0.125 & 1.399 & 28 & 1.050 & 1.084 & 18 & -0.100 & 1.549 \\
\hline 10 & 0.650 & 1.424 & 31 & 0.350 & 1.477 & 26 & 0.375 & 1.212 \\
\hline 12 & 1.600 & 0.955 & 32 & 0.725 & 1.300 & 27 & 0.275 & 1.260 \\
\hline 14 & 0.825 & 1.298 & 36 & 0.875 & 1.264 & 29 & 0.425 & 1.129 \\
\hline 15 & 0.800 & 1.399 & 38 & 1.425 & 0.843 & 30 & 1.100 & 1.032 \\
\hline 17 & 0.950 & 1.259 & 39 & 1.216 & 1.158 & 33 & 1.150 & 1.075 \\
\hline 19 & 0.575 & 1.214 & 40 & 1.050 & 1.036 & 34 & 0.725 & 1.300 \\
\hline 20 & 0.750 & 1.214 & 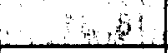 & & & 35 & 0.875 & 1.324 \\
\hline 21 & 0.350 & 1.459 & 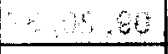 & $5, \mathrm{~b}$ & & $\lim 10 x$, & $\because$ & an \\
\hline 22 & 0.850 & 1.122 & 845 & 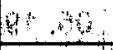 & 6,65 & $000 \mathrm{gal}$ & 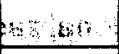 & 8 \\
\hline 24 & 1.000 & 1.240 & 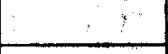 & 3 & 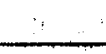 & 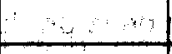 & $9 m$ & 0 \\
\hline 25 & 1.800 & 0.516 & $\therefore s$ & 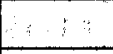 & & 40 & $\because ?, \ldots$ & 2 \\
\hline 37 & 1.400 & 0.955 & $E x$ & : & & & $\therefore$ & \\
\hline Subtotal: & 0.973 & 0.435 & Subtotal: & 1.120 & 0.392 & Subtotal: & 0.721 & 0.637 \\
\hline
\end{tabular}

La media global de las calificaciones otorgadas a las 17 afirmaciones agrupadas bajo el rubro "práctica de la investigación" indica un "acuerdo moderado", sólo interpretable en términos de cada afirmación, pero que hace resaltar cuatro calificaciones tan relativamente altas que acercan las afirmaciones respectivas a la categoría de lugares comunes entre los investigadores participantes, y una calificación cuya media es negativa (la única que en todo el instrumento indica claro desacuerdo colectivo).

En el grupo de 11 afirmaciones referidas a la "formación de investigadores" es en el que se concentra el mayor acuerdo relativo. Finalmente, en el grupo de 12 afirmaciones referidas a "la investigación como profesión", en el que se incluyeron las que se esperaba resultaran más polémicas por su contenido, más 
ideológico que descriptivo, la media global de las calificaciones apunta a la confirmación de la sospecha planteada.

\section{Representaciones de la estructuración del campo}

Para avanzar un poco más en la detección de rasgos del perfil ideológico proyectado por los investigadores encuestados, a través de convergencias y divergencias entre sus representaciones, se reagruparon las afirmaciones conforme a los nueve procesos de estructuración del campo académico de la comunicación postulados en el estudio, para relacionar los juicios "contenidos" en las afirmaciones (escritas por investigadores) con los juicios expresados sobre ellas por los sujetos (leídas por investigadores).

\section{CUADRO 2}

Afirmaciones por referencia a procesos de estructuración

\begin{tabular}{|l|l|l|}
\hline Procesos de estructuración & \multicolumn{1}{|c|}{ Afirmaciones } & Media \\
\hline Constitución de sujetos & 06,26 & 0.712 \\
\hline Conformación del habitus & $04,21,40$ & 0.525 \\
\hline Profesionalización & $13,17,18,31$ & 0.575 \\
\hline Institucionalización social & $03,08,09,20,37$ & 0.625 \\
\hline Institucionalización cognoscitiva & $05,19,22,25,30,32,36$ & 1.007 \\
\hline Especialización de la producción & $01,10,11,16,24,35,38,39$ & 0.983 \\
\hline Autorreproducción del campo & $14,15,23,27$ & 0.812 \\
\hline Legitimación social & $07,12,28,33$ & 1.100 \\
\hline Asimilación/acomodación & $02,29,34$ & 0.891 \\
\hline
\end{tabular}

A partir de esta nueva agrupación referencial se analizaron los comentarios escritos por los investigadores acerca de cada afirmación.

En cuanto a los procesos de constitución de los sujetos (afirmaciones 06 y 26$)^{2}$ pueden identificarse dos juicios encontrados: los que valoran positivamente el

2. (06) "Existen miembros de nuestra comunidad cuya identidad profesional está muy cercana al hombre de letras; otros comparten más de cerca su identidad como politólogos, sociólogos, historiadores, semiólogos, psicólogos clínicos y sociales, educadores, ingenieros, etc." (Abraham Nosnik, 1988). (26) "Generar conocimiento y transformar la sociedad son proyectos cuya realización exige la recurrencia a principios de acción distintos y muchas veces opuestos; los factores básicos para la organización del trabajo y para la definición de las operaciones que conduzcan hacia objetivos de uno u otro género, suponen lógicas diversas, difícilmente 
carácter "híbrido" de la identidad profesional de los investigadores de la comunicación ("es propio de la comunidad y un posible factor de enriquecimiento") y los que lo consideran un factor de subdesarrollo ("este problema se debe a la indefinición clara [sic] de nuestro campo de estudio"). En resumen, "somos una fauna muy heterogénea" pues "el estudio de la comunicación como fenómeno es multidisciplinario" y "creo que se puede y debe trabajar de manera conjunta en un proceso de cambio [social]".

Por lo que toca a los procesos de conformación del habitus, las tres afirmaciones agrupadas al respecto $(04,21 \text { y } 40)^{3}$ suscitan "tensiones" ideológicas muy fuertes en cuanto a la orientación básica de la investigación. De ahí que sea éste el grupo con menor promedio de "acuerdo": no hay consenso sobre la "investigación militante" ("el peligro de esta situación es la pérdida de los contenidos básicos de la especialidad en aras de una formación partidista"), la "investigación de frontera" ("ésta es la esencia de la investigación") o la "investigación dependiente" ("aunque parezca que es una afirmación ideologizada, pienso que tiene razón en no descartar ni lo subjetivo del estudio ni lo macro, pero sin perder tampoco lo objetivo y lo micro a fin de lograr una visión más integrada de lo comunicacional dentro de lo sociocultural"). Muchos de los comentarios tienden a relativizar o a matizar las afirmaciones ("Hay un rango muy amplio de diferencias") y uno de ellos resume el desacuerdo en que "hay muchos tipos de investigación, desde la seria, teórica, bien formulada, hasta la que está al servicio de los políticos: problema ético".

Los comentarios referidos a las cuatro afirmaciones $(13,17,18 \text { y } 31)^{4}$ agrupadas en relación con los procesos de profesionalización de los investigadores,

conciliables; los sujetos que realizan esos proyectos a través de estos trabajos adquieren identidades sociales distintas" (Raúl Fuentes, 1992).

3. (04) "La investigación de la comunicación se ha asumido en América Latina como un instrumento para mejorar la calidad de la vida y como un modo de procurar la justicia, promover el cambio y denunciar la manipulación. De hecho, la implicación política para muchos precede y guía la práctica de investigación hasta el punto que, en muchas ocasiones, los roles del académico y del político se vuelven intercambiables" (Elizabeth Lozano y Josep Rota, 1990). (21) "Una investigación de comunicación no sirve para confirmar lo que sabemos, sino para descubrir algo que no sabemos. Una investigación que no nos sorprende es una mala investigación" (Eliseo Veron, 1991). (40) “A la intención ataráxica de quienes desearían nos ocupásemos a tiempo completo de la semiología del videoclip, hemos de enfrentar la lucidez intelectual y política de quien también piensa la totalidad. A la astucia de quienes pregonan el final de la historia para que ya no nos ocupemos en hacerla, hemos de oponer propósitos de participación activa en el reordenamiento del mundo" (Antonio Pasquali, 1992).

4. (13) "Quien se dedica a la investigación tiene que tener una carga horaria docente mínima. Esto no quiere decir que no tengan que dar clases los investigadores; quiere decir que tiene que ser la menor parte de su tiempo la que dediquen a la enseñanza y la mayor parte de su tiempo a la investigación, que es una actividad que consume mucho tiempo y los resultados, desafortunadamente, no se ven de inmediato" (Pablo Arredondo, 1992). (17) "La cientificidad de una investigación, lejos de estar garantizada por el uso de ninguna técnica ni de programas de computadora, se funda en el tipo de preguntas que se pueden plantear, en el tipo de relaciones complejas que se pueden establecer solamente a partir de aquéllas" (Jorge 
dejan ver con mayor claridad los polos del desacuerdo: en cuanto a la articulación investigación/docencia, algunos están "de acuerdísimo, no más comentarios" con que los investigadores dediquen "la menor parte de su tiempo" a la enseñanza, pues "sólo en la medida en que el investigador puede dedicarse plenamente a su trabajo, es que la docencia adquiere sentido". Pero para otros, "planteado así, no se puede avanzar. No se trata de proporciones o cantidades. El punto es la identidad del académico y el cómo se entienda la docencia. Ésta es la puesta en común de conocimiento y la generación de conocimiento nuevo $\mathrm{y}$, entonces, es un proceso de investigación también". Igualmente se advierte que "se requiere ligar docencia-investigación [porque] hay riesgo de perder a los nuevos cuadros de investigadores". En relación con la "cientificidad" por las preguntas o por las técnicas, se dice que "habría primero que definir lo científico y lo que no lo es", pero también que "no basta que las preguntas sean relevantes y trascendentes", que deben estar "acompañadas de los procedimientos de comprobación" y que "la cientificidad abarca todo el proceso, no puede darse por sólo una parte del mismo".

Sobre si el "progreso" en investigación depende de ocupar puestos administrativos, predomina el desacuerdo, aunque el sentido de rechazo al contenido de la afirmación es casi unánime. Algunos aceptan el hecho, si bien lo valoran negativamente ("Así es en la mayoría de los casos. Quien quiere destacar como investigador, no sólo en nuestro campo, requiere de espacios públicos y políticos para hacerlo. Quizá esto se deba a la poca relación que existe entre los investigadores y el campo laboral”), y otros lo niegan (“iNo! Los investigadores de mayor prestigio aquí y fuera del país creo que son los que no han sido atrapados por la administración"), pero nadie lo defiende explícitamente, porque "no es así y no debe ser así, aunque hay una mentalidad generalizada de que no hay otra forma. Eso es lo que hay que demostrar, que sí la hay y que progresar en investigación debe evaluarse académica/científicamente, no políticamente como se hace ahora".

Probablemente la afirmación más "agresiva" hacia los sujetos es la que sostiene (31) que "uno de los principales obstáculos para la investigación social en México es la baja calificación de la mayoría de los investigadores". El mínimo grado de acuerdo alcanzado (0.333) proviene básicamente de la oposición entre algunas reacciones de aceptación ("en el contexto de una disciplina centrada en la atención a la cada vez mayor demanda estudiantil por la licenciatura, es explicable”) y una mayoría de elusión (“ien qué sentido descalificación?"; “ipor calificación se entienden grados académicos o capacidad?"). También hubo

González y Rossana Reguillo, 1992). (18) "El camino del progreso para los mejores investigadores pasa inevitablemente por los puestos administrativos, que implican un mayor reconocimiento tanto material como de prestigio dentro de la comunidad académica. Paradójicamente, para progresar en la carrera de investigación, hay que dejar de hacer investigación" (Larissa Lomnitz, citada en Fuentes y Sánchez, 1989). (31) "Uno de los principales obstáculos para la investigación social en México es la baja calificación de la mayoría de los investigadores” (Raúl Fuentes y Enrique Sánchez, 1989). 
comentarios para matizar el desacuerdo con la priorización de otros factores: "la dificultad mayor es la falta de apoyo"; "es la falta de proyectos colectivos y fundamentalmente de dinero"; "creo que hay muchos factores que se entrelazan: carencia de recursos, confusión teórico-metodológica, falta de apoyo institucional, aislamiento, etc. etc.". Uno de los sujetos ("fatalista") anotó que los investigadores "no son peores que los políticos, empresarios, obreros, campesinos, sacerdotes, profesores, deportistas y otros especímenes de nuestra triste realidad. Basta verlo a la vuelta de cada esquina". Otro ("optimista"), en cambio, señaló su acuerdo, pero "con muchas y valiosas excepciones".

Cinco afirmaciones $(03,08,09,20 \text { y } 37)^{5}$ se relacionan con los procesos de institucionalización social u organización del campo académico. La primera de ellas, relativa a la (des)articulación investigación-posgrados, suscitó un grado alto de acuerdo, pero también la repetida reserva de que "no se puede generalizar". Cinco de los sujetos se sintieron obligados a exceptuar explícitamente a su institución de la situación descrita. La segunda, referida a la "intercomunicación" del campo académico de la comunicación en América Latina, es la que obtuvo la calificación más alta de desacuerdo, sobre todo por la respuesta de los académicos de las instituciones públicas y de las capitalinas, según la prueba $t$ de Student aplicada. Muchos de los comentarios son aún más elocuentes que la calificación de desacuerdo: "existe aislamiento de los investigadores, repetición de trabajos, desconocimiento de los resultados"; "En México es realmente escasa la intercomunicación"; "faltan mecanismos para lograr comunicación, cooperación y publicaciones"; "pienso que esta interactividad está limitada a un grupo pequeño de investigadores".

Este factor, la articulación "social" del campo académico a escala latinoamericana (representada positivamente en la afirmación por un norteamerica-

5. (03) "Los posgrados en comunicación en México no son instancias de investigación que alimenten a programas de formación, sino que surgen de la demanda y la estructura escolar. Los programas de maestría son propuestas que, viniendo desde la docencia, tienen a la investigación más como un problema que como un insumo" (Reunión de Posgrados e Investigación, 1989). (08) "Una de las más llamativas características de las investigaciones en materia de comunicación en América Latina - un poco en contraste con lo que ocurre en Europa y en otras partes del mundo- es la notable intercomunicación que existe entre los investigadores, los proyectos de investigación cooperativa y la conexión entre diversas organizaciones, institutos, publicaciones y facultades" (Robert White, 1989). (09) "La panorámica del campo de la investigación de la comunicación en México presenta una aparente paradoja: en condiciones de crisis (social) ha crecido y consolidado bases, se ha diversificado y fortalecido" (Raúl Fuentes y Enrique Sánchez, 1992). (20) "Entre 1985 y 1990 se han sentado bases muy importantes para una mayor profesionalización de la investigación mexicana en comunicación y se han creado diversas oportunidades de interrelación de los esfuerzos de investigadores antes aislados. Asimismo, se han multiplicado las posibilidades de desarrollo de la indagación sistemática en diversas regiones del país, todo lo cual ha contribuido a crear una nueva configuración, esperamos que más promisoria, del campo" (Raúl Fuentes y Enrique Sánchez, 1992). (37) "La visión más amplia del país, que no se puede centralizar toda porque eso es reducirla y no se puede atender desde el centro únicamente, porque eso es deformarla, es una necesidad muy importante. La comunicación está en todo el país y con muchas diferencias; entonces, hay que investigarla desde todo el país" (Raúl Fuentes, 1992). 
no) y su reconocimiento (negativo) por parte de los investigadores mexicanos (incluyendo a varios de los que mantienen los vínculos latinoamericanos más fuertes), aporta uno de los indicios más interesantes del perfil ideológico que se intenta construir.

Algo similar sucede con la afirmación referida al crecimiento y la consolidación de la investigación de la comunicación en México en un contexto de crisis, que obtuvo una calificación media apenas positiva. Hay quienes están de completo acuerdo y comentan que "es la misma situación de crisis la que sirve de acicate para buscar salidas a la misma por el recurso de la investigación" o que "no sólo la investigación de la comunicación, sino muy especialmente la creación literaria, y no sólo en México sino en todo el mundo". Pero la mayoría expresa dudas en uno u otro sentido: "se ha diversificado pero no consolidado. Existen graves lagunas en muchos campos"; "al contrario, se está restando apoyo a proyectos de investigación"; "Pocos jóvenes se han incorporado a la investigación, con relación a las camadas de egresados de la carrera"; "Mucho de lo que se hace como 'investigación' no cumple las reglas mínimas de calidad teórica y metodológica. En este sentido, hay muy poca investigación en México".

En el mismo sentido, aunque con una calificación media de mayor acuerdo, se orientan los comentarios a la afirmación sobre la profesionalización y regionalización de las prácticas de investigación, con respecto a lo cual "hay que matizar mucho, porque cada vez hay más investigadores que abandonan el área y se van a otras y también hay menos financiamiento", o que todo "depende del parámetro de juicio: aquello del vaso medio lleno o medio vacío". El único comentario que acompaña a una calificación de acuerdo pleno con la afirmación señala que "ojalá así continúe y no sea otra moda más". Es más claro, en cambio, el sentido del desacuerdo: "la investigación sigue siendo en lo fundamental un acto personal y casi aislado. Los espacios nuevos se siguen cooptando por pequeños grupos cerrados". Los comentarios a la afirmación sobre la "descentralización" de la investigación sólo refuerzan el sentido ( $¿$ "políticamente correcto"?) del acuerdo.

Por lo que toca a las siete afirmaciones que se agruparon en referencia a los procesos de institucionalización cognoscitiva, hay algunos sentidos claramente divergentes en las interpretaciones manifiestas en los comentarios. En cuanto a la prevalencia de las "modas" en la investigación de la comunicación (05) ${ }^{6}$ hay acuerdos francos ("cuestión que no sólo ocurre en la comunicación, sino también en otras ciencias sociales"), intentos de "profundización" del diagnóstico ("muchos sí y la razón puede ser porque no había hasta hace poco investigadores realmente capacitados como tales"), desacuerdos con la generalización ("varía según el lugar y los individuos") y una asociación discursiva que se presenta en dos casos: "habría que matizar o precisar: a veces la 'moda' significa coyuntura"

6. (05) “Gran parte de los trabajos en investigación en comunicación obedecen más a modas, que tan pronto suscitan el entusiasmo como el olvido de los investigadores" (Raúl Trejo, 1988). 
y "una cosa son 'modas' y otra 'coyunturas', no sé a cuál de las dos quisieras referirte [sic]". Tal referencia a las "coyunturas" desplaza .el eje de atención de los enfoques teórico-metodológicos (que se adoptan y abandonan como modas) a la articulación de la investigación con las cambiantes situaciones políticas de los sistemas de medios masivos y sus relaciones con el Estado, preocupación explícitamente formulada por la Asociación Mexicana de Investigadores de la Comunicación (AMIC) en los años ochenta.

Las afirmaciones 19 y $32^{7}$ guardan entre sí una estrecha correlación tanto semántica (sentido de la enunciación) como estadística (índice de 0.451 entre las respuestas a una y otra). Ambas tienen como referentes los cambios tecnológicos y políticos de los procesos de comunicación social y los criterios "neoliberales" para apoyar o no su estudio, aunque una enfatiza la oposición macro-micro y la otra el binomio comunicación-información. En ninguno de los dos casos parece haber posiciones claramente definidas de acuerdo o desacuerdo en los comentarios, probablemente porque la "densidad" referencial de las citas exigía mayor contextualización ("depende del espacio donde se da el debate"), aunque algunos comentarios adoptan el sentido sugerido por las propias afirmaciones ("a veces no se tiene conciencia de la racionalidad tecnocrática desde la que se estudia la comunicación ni de su reduccionismo a los medios. Hace falta impulsar la racionalidad comunicativa que define los medios en función de los fines y significados a lograr"; "ciertamente veo una tendencia a realizar estudios mucho menos totalizadores y más puntuales, aunque no necesariamente desligados de una realidad más amplia").

En una escala más concreta del juicio sobre los cambios cognoscitivos en la práctica de la investigación se sitúan las afirmaciones 25 y $30 .^{8}$ Ambas apuntan

7. (19) "Cuando mayor solidaridad va existiendo entre la producción de comunicación y la producción y reproducción social, los estudios macro-sociológicos cada vez se producen en menor cantidad. Desde el comienzo de la década de 1980 se inicia una inundación de estudios micro-sociológicos, centrados en investigar los efectos prácticos que en el consumo de información tiene la incorporación de tal o cual tecnología. Ciertamente que estos estudios, centrados en los efectos de mercado, tienen utilidad práctica e incluso interés teórico. Pero existe otro nivel de análisis de los efectos, aquel en el que se relacionan con sus causas y con sus consecuencias, que en la práctica han dejado de financiarse" (Manuel Martín Serrano, 1992). (32) "Pareciera haber una insistencia generalizada en tratar de reducir el problema de la comunicación al de la información. Esto en parte se explica por el desarrollo de las nuevas tecnologías de información y por el deslumbramiento social que causa su potencial. Pero sobre todo tiene su origen en una concepción tecnocrática del proceso comunicativo, que a su vez obedece a una racionalidad enfocada en los medios y no en los fines. Es la tecnicidad de la información y no su representatividad sociocultural la que ha estado predominando en los debates recientes" (Guillermo Orozco, 1992).

8. (25) "Poco a poco, haciendo de lado los intentos 'esencialistas' de explicación, entendemos los fenómenos y procesos de comunicación masiva como complejos y multidimensionales, que operan en sociedad a diversos niveles de generalidad social, con diversos tipos de articulación y por lo tanto de interacción con la economía, las estructuras de poder, la cultura y la estructura y movimientos sociales" (Raúl Fuentes y Enrique Sánchez, 1992). (30) "La investigación de los procesos de comunicación puede ser entendida como parte de un proceso cultural complejo que implica encuentros diversos entre esquemas de clasificación de la 
al reconocimiento de la complejidad y multidimensionalidad de los procesos de comunicación social. La primera es la que alcanzó la puntuación más alta de todo el instrumento, en un acuerdo prácticamente completo por parte de los sujetos, pues "esto ha permitido comprender mejor el proceso de comunicación aunque muchas veces nos ha lanzado hacia otras áreas disciplinarias, perdiendo el punto de vista de la nuestra" o bajo la consideración de que "por ello es necesario adoptar estrategias transdisciplinarias para el estudio de la comunicación". La segunda es, a su vez, la que menos comentarios suscitó (sólo siete), incluyendo un "de acuerdo, aunque no con la terminología empleada".

Las afirmaciones 22 y $36^{9}$, finalmente, refieren a juicios sobre la escala más inmediata de la práctica de los sujetos investigadores, incluyendo la construcción gramatical en términos de "nosotros", en cuanto a las tensiones "ensayismoempirismo" y "medios influyentes-públicos activos". Los grados de acuerdo con ambas afirmaciones son medios, y los comentarios permiten clarificar el sentido de las divergencias: algunos sujetos, nuevamente, combaten la generalización ("aquí el plural 'estamos' no sirve. Hay quien vive en la empiria superficial y hay quien no sale del ensayismo"). Otros están de acuerdo, pero muestran reservas ("es una tendencia emergente, pero que falta todavía para que se haga extensiva"), y algunos más expresan desacuerdo ("lo dudo, ¿de quién se está hablando?") y apuntan hacia las causas ("la realidad de las condiciones de trabajo de los comunicadores a veces no permite abandonar el ensayismo"; "la política de los puntitis, pilones, etc. [sic] obliga muchas veces al ensayismo").

La mayoría de los comentarios connotan negativamente el "ensayismo", como la afirmación, pero dos de los sujetos lo reivindican: "sigo pensando en la bondad de los ensayos, cuando son sugerentes y ricos en determinaciones conceptuales. Frente al ensayismo están las tautologías de carácter cuantitativista"; "el ensayo seguirá siendo necesario para formular propuestas originales como ocurre, por ejemplo, en la investigación literaria y humanística en general". En cuanto al binomio "medios influyentes-públicos activos", se presentan desde el desacuerdo tajante ("es abaratar mucho las diferentes perspectivas, que a diferencia del juicio apresurado del autor de la cita, proponen argumentos respetables") hasta el acuerdo por razones "positivas" ("en el pasado se ha caído en estos polos. Sin embargo, considero que ya hay una mayor conciencia en torno a esto") o "negativas" ("cierto, no damos para más"). Uno de los sujetos resumió

realidad que tienen espesor y densidades no sólo diferentes sino a veces contrapuestas. Son definiciones de la realidad que se confrontan a diario y luchan desde posiciones desniveladas por el poder, por las clases" (Jorge González y Rossana Reguillo, 1992).

9. (22) "Estamos en una transición muy sana del 'ensayismo' al uso sistemático y más riguroso de metodologías y técnicas de investigación para sustentar con evidencia concreta, empírica, los desarrollos conceptuales" (Enrique Sánchez y Pablo Arredondo, 1988). (36) "En virtud de modelos teóricos y diseños de investigación inadecuados, algunos de nosotros hemos 'sobre-enfatizado' las influencias más o menos directas de los medios sobre sus públicos, mientras otros han exagerado la 'libertad' y actividad de los últimos” (Raúl Fuentes y Enrique Sánchez, 1992). 
en una frase el sentido de la afirmación: "parte de un péndulo que necesita encontrar su momento de síntesis".

El siguiente grupo de afirmaciones, las referidas a los procesos de especialización de la producción del campo académico de la comunicación, aporta nuevos indicios de los "ejes de sentido" sobre los que los investigadores interpretan el propio campo y sus posiciones en él desde la dimensión cognoscitiva de sus prácticas y sus juicios. En lo referente a las articulaciones sociales de la investigación, hay un alto grado de acuerdo en la "determinación social" de la investigación social (afirmación 01 ) ${ }^{10}$ pues "en lo social la investigación es una actividad que debe justificarse y dicha justificación se hace a partir de las necesidades sociales o humanas necesitadas de solución"; y "esta 'compleja interacción' entre investigación y estructura social puede ser resultado de un diálogo entre procesos sociales y exploraciones epistemológicas".

En una escala metodológica (afirmación 10), ${ }^{11}$ los desacuerdos con la función "transformadora" de la investigación provienen tanto de juicios contrarios sobre la orientación ("no creo que toda investigación implique lo planteado") como sobre la viabilidad ("de acuerdo, aunque es una posición un tanto utópica. En la realidad se da de otro modo, más autoritario"). El grado de acuerdo global resultante es relativamente bajo, sobre todo porque se cuestiona la articulación "militante" o "comprometida" de la investigación sugerida por la afirmación ("me parece una afirmación extrema y restrictiva al estilo de 'sólo hay conocimiento si...").

Este cuestionamiento se refuerza notablemente en los comentarios a la afirmación $16,{ }^{12}$ que obtuvo una calificación de acuerdo más baja y que guarda con la afirmación anterior un índice de correlación estadística relativamente alto (0.506). En este caso, los comentarios son más categóricos en la divergencia ("considero esta afirmación un tanto demagógica"), aunque por la referencia a la AMIC como sujeto de la afirmación el desacuerdo se canaliza en muchos casos hacia un juicio sobre la asociación ("sería muy bueno, pero siento que AMIC necesita crear propuestas más sólidas, puntuales, fundamentadas, viables, etc. etc.”). Son especialmente significativos los comentarios de tres ex presidentes de

10. (01) "La investigación científica, especialmente la que se ocupa de lo social, no se autogenera a partir de finalidades "puras", por ejemplo teóricas, sino que surge y se desarrolla en una compleja interacción con las estructuras fundamentales y las subestructuras pertinentes de la sociedad" (Enrique Sánchez y Pablo Arredondo, 1988).

11. (10) "Lo que se requiere para entender los medios masivos de comunicación y su influencia en los auditorios no es más investigación per se, sino un cierto tipo de investigación. Una investigación donde investigador y objeto de análisis estén comprometidos con una finalidad de transformación, donde la generación de conocimiento nazca de una reunión dialéctica entre sujeto cognoscente y objeto de estudio y donde el conocimiento obtenido responda a necesidades específicas de un sector del auditorio para el uso social de un medio de comunicación" (Guillermo Orozco, 1988).

12. (16) "Es de la más alta prioridad ciudadana que la AMIC y la débil sociedad civil que existe en el país, colaboren en la producción de un nuevo programa de gobierno que oriente el funcionamiento de las estaciones de televisión pública hacia la atención de los conflictos centrales que atoran nuestro proyecto de desarrollo nacional" (Javier Esteinou, 1988). 
la propia AMIC: "No sé si AMIC pueda por sí misma hacer nada, pues carece de organicidad y organización y su capacidad de convocatoria y peso político se han diluido."; "la AMIC no tiene representatividad ni estructura suficientes para ello. No es su tarea hacer propuestas para gobernar"; "Se caería en esa subordinación a la coyuntura que tanto nos desgastó". En cuanto a las articulaciones sociales de la investigación, el grado de acuerdo asignado a la afirmación $38^{13}$ en relación con el impacto medible de los medios sobre las identidades culturales latinoamericanas, sólo es cuestionado en cuanto a la posibilidad o pertinencia de lo medible.

En otros aspectos de la especialización de la producción, los comentarios a la afirmación sobre la investigación y las prácticas de la comunicación organizacional (afirmación 11), ${ }^{14}$ tienden a aceptar el acercamiento, pues "este y otros aspectos merecen procesos de reflexión para encontrar puntos de acuerdo" y porque "la investigación en comunicación organizacional está urgida de ese diálogo con la academia: necesita consolidar marcos teóricos menos pragmáticos y descriptivos". Sin embargo, hay algunas reservas en cuanto a la coincidencia "en que hay menor polarización y búsqueda de puntos de interés, pero esto no sólo se circunscribe al área de la comunicación organizacional", y en cuanto a que "la investigación no tiene que ser necesariamente propositiva. La denuncia no surge porque así se lo proponga el investigador, sino por las condiciones reales de América Latina".

Curiosamente, la afirmación $35,{ }^{15}$ que postula el distanciamiento entre la investigación y la práctica profesional de la comunicación, tiene un nivel de acuerdo más bajo que la anterior, pero comentarios en sentidos muy divergentes (acuerdo, pues "la investigación en México se ha dedicado sobre todo a satanizar las prácticas profesionales"; desacuerdo, "ipero si una es consecuencia de la otra!"; desplazamiento, "no en todos los casos"; "tiende a disminuir"; duda, "depende de lo que se entienda por práctica profesional"; "no sé, habría que preguntar a las agencias de información de mercado"; "¿habrá alguien realizado la crítica al campo profesional?").

13. (38) "Correspondería a la investigación emprender por primera vez un trabajo concreto y muy necesario, consistente en determinar qué impacto medible ha venido ejerciendo la incorporación progresiva de canales, tecnologías y medios de comunicación en la formación y evolución de las identidades culturales de los países latinoamericanos" (Antonio Pasquali, 1992).

14. (11) "El desarrollo de la profesión en el ámbito institucional y la disminución de la polarización de las posiciones, así como el encontrar puntos comunes de interés, a pesar de las diferencias, pueden llevar a una apertura en el ámbito académico que permita hacer una crítica fundamentada (más allá de la mera denuncia de los males del capitalismo) y propositiva a la comunicación organizacional y a un acercamiento de los profesionales en el campo a las universidades, para reflexionar seria y críticamente sobre las implicaciones sociales de esta profesión" (Pablo Casares, 1992).

15. (35) "La investigación sobre comunicación colectiva ha estado distanciada de las realidades de la práctica profesional en nuestro país” (Raúl Trejo, 1988). 
Sobre la afirmación $24,{ }^{16}$ referida a la "falta" de una publicación especializada en comunicación, el acuerdo relativamente alto no excluye juicios críticos ("las que hay son expresiones de feudos o grupos"; "el problema es tener buenas publicaciones"; "lo que hace falta es que las existentes circulen mejor y paguen las colaboraciones").

La afirmación $39^{17}$ es la que plantea con mayor precisión la cuestión de la "especificidad" disciplinaria de los estudios de comunicación. El acuerdo relativamente alto con que el "objeto 'comunicación' en sí mismo ha sido dejado de lado" en gran medida en la investigación, y el sentido de algunos de los comentarios, tienden a confirmar la tendencia a la inespecificidad disciplinaria del campo, tanto en los sujetos que señalan su acuerdo como en los que están en desacuerdo con la afirmación: "ciertamente, lo específico de la comunicación pasa desapercibido, es ignorado, no inquieta, no preocupa, no se contempla como problema de investigación; los trabajos se orientan a lo que aparece y es evidente del fenómeno, falta profundizar en él"; "concibo a la comunicación a la manera de una paradisciplina. Esto es, sustantivada por otros campos del saber"; "creo que es necesario asumir que las ciencias de la comunicación sólo pueden constituirse en las ya existentes. Su especificidad y su dificultad están en el enfoque y en la articulación".

Hasta aquí, han quedado claras algunas de las principales divergencias en las representaciones sostenidas por los investigadores sobre algunas características del campo, las cuales son reconfirmadas en los comentarios anotados con respecto a las cuatro afirmaciones que se agrupan en referencia a los procesos de autorreproducción del campo. La afirmación $14^{18}$ regresa al tema del "abandono" del denuncismo o la investigación militante en contraposición a la "ciencia". Los comentarios, tanto de acuerdo como de desacuerdo, van en su mayoría en el sentido de revalorar, más que de desechar el compromiso social ("de acuerdo, pero sin perder en el fondo el espíritu de cambio"; "los graves errores cometidos

16. (24) "Sigue haciendo falta una publicación especializada en comunicación, así como las hay en economía, sindicalismo, administración pública, relaciones industriales o tantas otras disciplinas" (Raúl Trejo, 1988).

17. (39) "Dentro de este campo de relaciones y prácticas sociales que se ha llamado 'investigación de la comunicación en México' puede decirse que, en gran medida, el objeto 'comunicación' en sí mismo ha sido dejado de lado - con pocas pero valiosas excepciones- para abocarse los investigadores a desentrañar las múltiples dimensiones y niveles de la operación social de los medios de difusión y sus productos, así como sus articulaciones con los procesos sociales más amplios, lo que ha significado partir de - y con frecuencia 'llegar a'- enfoques disciplinarios también más amplios, desde las ciencias sociales" (Raúl Fuentes y Enrique Sánchez, 1992).

18. (14) "Es preciso revisar, en el caso latinoamericano, las experiencias de investigacióndenuncia, investigación-acción, investigación participante, entendiéndolas como alternativas marcálas por la coyuntura de resistencia al autoritarismo de los años 70 y 80 , pero que se desgastaron por las distorsiones metodológicas emprendidas por investigadores convertidos ingenuamente en misioneros de causas nobles, volviéndose cómplices de un desvirtuamiento de la actividad científica. Por eso mismo, perdieron la credibilidad y dejaron de contribuir, como científicos, a la construcción de un nuevo orden de la comunicación" (José Marques de Melo, 1991). 
no significan negar una propuesta cada vez más necesaria"; "creo que hay muchas acciones de estos 'misioneros' que requieren ser estudiadas y evaluadas, y sobre todo entendidas en el contexto en que se dieron. Aprendamos de nosotros mismos").

La afirmación $23^{19}$ de alguna manera coincide en el sentido de revalorar los aportes latinoamericanos pioneros sobre todo en la docencia con las nuevas generaciones. Aunque el índice de acuerdo es mayor, los comentarios son menos elocuentes: constatan que "hay poco interés sobre el tema, o falla en la manera de transmitirlo", que "esto es lamentable y lo haría extensivo a los pioneros del campo a nivel internacional" y que "al respecto apenas se está construyendo un capital académico". Hay quien opina que "América Latina ha dado mucho en materia de comunicación y falta aún mucho por dar” y quien, por el contrario, juzga que "poco trascendente se ha hecho en América Latina. Hay mucha paja, copia y relumbrón".

En cuanto a la percepción de los sujetos sobre la investigación no-académica, la afirmación 15,20 que la considera limitada, obtiene un grado medio de acuerdo, pero a partir de dos posiciones polarizadas: algunos sujetos coinciden en que la investigación que realizan las empresas privadas "busca sólo ampliar conocimientos en torno a la venta de mercancías o posicionamiento de empresas", aunque otros ven "una tendencia a ampliar estos ámbitos (ideologías profesionales/rutinas productivas) buscada por ellos", o que "la preocupación se ha centrado, más que nada, en mejoras metodológicas. Hay poco tiempo para la reflexión y el análisis, pero sí se realiza en algunas de ellas". Otros comentarios reconocen que hay estudios "también de las estrategias discursivas y de la competencia profesional, sólo que estos estudios son empíricos y, sobre todo, no divulgados" o que basta con "ver que las programaciones y contenidos han dado un vuelco y se debe a que sí investigan otras cosas que no sólo el marketing." Alguno más confiesa: "no conozco realmente cómo están estas empresas".

La afirmación $27^{21}$ regresa a la consideración del carácter de la AMIC. Aunque en las respuestas no se encuentra un índice significativo de correlación estadística con la afirmación $16(0.067)$, el grado de acuerdo alcanzado es similarmente bajo (0.297). Aparte de dos sujetos que se abstienen porque "des-

19. (23) "No se ha extendido plenamente en nuestro continente la conciencia sobre el papel desempeñado por los investigadores y los centros de investigación latinoamericanos en la producción de conocimiento científico sobre la comunicación social. Más bien se constata un relativo desconocimiento de las nuevas generaciones sobre el pensamiento construido por nuestros pioneros en el área" (José Marques de Melo, 1988).

20. (15) "Las empresas privadas de comunicación se han preocupado poco por estudiar la naturaleza de su trabajo. El único seguimiento regular que han tenido es el análisis de mercados y públicos" (Raúl Trejo, 1988).

21. (27) "Ha habido intentos serios, de gente seria, por hacer que la AMIC sea un espacio que aglutine a investigadores, que promueva la investigación, al gremio, la presencia social y política. Pero hay muchas limitaciones estructurales que no dependen de la voluntad de qujen está al frente, o de las iniciativas de la gente. El hecho de que sea una asociación de investigadores en lo particular, le resta fuerza" (Carlos Luna, 1992). 
conozco la historia de la AMIC", los comentarios se polarizan entre quienes coinciden en que "el hecho de que sea una asociación de investigadores en lo particular, le resta fuerza" y quienes niegan que haya "habido intentos serios" por consolidar a la AMIC como una asociación académica.

En conjunto, los comentarios (vertidos entre 1992 y 1993) muestran una imagen muy desfavorable de la asociación entre los sujetos, independientemente del acuerdo o desacuerdo con la afirmación citada, tanto entre quienes son (o han sido) miembros de ella ("creo que los 'intentos' han sido realmente iescasos! Desde un principio la AMIC se manejó más dentro de lo político que de lo académico. Poco se hizo para trabajar en lo interior") como entre quienes no lo son ("los intentos serios son relativos. La verdad es que desde fuera se nota que falta una integración real de los que son investigadores de la comunicación. Parece que hay deseo de aglutinar gente que presente cualquier trabajo de investigación sin ser investigador que justifique con actividad y práctica el nombre. No habrá desarrollo de esa organización si no es rigurosa en la selección de sus miembros"). Sin embargo, son muchos los sujetos (miembros y no miembros de la AMIC) que reivindican la viabilidad y la conveniencia de una asociación de individuos: "Considero que el proyecto de la AMIC es viable como asociación de investigadores, y es importante mantener ese espacio. Tal vez [haya que] intentar formas de organización y participación diferentes"; "Habría en todo caso que enfatizar su actividad académica, sin olvidar su responsabilidad política. El hecho de que sea de investigadores es parte de su fuerza"; "su escasa fuerza no se debe a su identidad de 'investigadores', sino a que no los ha podido aglutinar".

En relación con los procesos de legitimación social se agruparon cuatro afirmaciones y de los nueve "grupos" formados en torno a los procesos de estructuración del campo académico, es éste el que alcanzó una calificación conjunta de mayor acuerdo (1.100). Sin embargo, en su interior pueden distinguirse dos subgrupos de dos afirmaciones cada uno, pues la 07 y la 28 tienen que ver con la insuficiencia del conocimiento generado sobre el entorno social (económico-político), ${ }^{22}$ mientras que la 12 y la 33 se refieren a acciones estratégicas del propio campo. ${ }^{23} \mathrm{El}$ relativo consenso alcanza un grado mayor en el

22. (07) "Queda un muy largo trecho por recorrer para lograr entender las dimensiones políticas de los diversos medios de difusión y es mucho lo que se ignora todavía sobre el papel real de los medios en los procesos de acumulación de capital" (Raúl Fuentes y Enrique Sánchez, 1992). (28) "Las nuevas tecnologías de comunicación no sólo ocupan el lugar central de un reto industrial; están en el corazón mismo de las estrategias de reorganización social de las relaciones entre el Estado y el ciudadano, los poderes locales y centrales, los productores y los consumidores, los patronos y los trabajadores, los enseñantes y los enseñados, los expertos y los ejecutantes. En este contexto de mutaciones científicas y tecnológicas han surgido nuevos actores históricos, tanto en el campo de la industria y del mercado como en el de las estrategias de resistencia social, tanto en el 'primer' mundo como en el 'tercero"' (A. y M. Mattelart, 1987).

23. (12) "Los productos de la investigación social deben ser tan ampliamente difundidos y utilizados como sea posible. Primero, dentro de la propia comunidad académica y luego entre 
segundo subgrupo que en el primero, lo cual podría indicar que son más fácilmente compartidos los propósitos (éticos) de la investigación que los diagnósticos sobre su orientación.

Algunos investigadores no están muy de acuerdo en que el conocimiento disponible acerca de las dimensiones políticas y (macro) económicas de los medios sea insatisfactorio ("creo que este aspecto es suficientemente conocido, en términos estructurales, aunque faltarían tal vez más investigaciones empíricas realizadas desde la disciplina económica"), aunque otros coinciden con el autor de la cita ("es poco lo que conocemos y, ahora, es importante introducir a las nuevas tecnologías, cuyo papel parece ser muy relevante en la acumulación de capital. Asimismo, necesitamos nuevas categorías que nos permitan comprender los procesos de globalización en lo que se refiere a este punto"), mientras que otros más subrayan las (des)articulaciones con la inespecificidad disciplinaria del campo: "considero que este aspecto ha sido un objeto de estudio ampliamente privilegiado, pero mal construido porque se ha centrado en los medios y en la macroestructura sin incorporar dimensiones que enriquezcan el conocimiento de la dinámica social en el sentido de la apropiación y la producción simbólica".

Con respecto a la afirmación 28 , algunos de los sujetos comentan que "lo que está en el centro de las 'estrategias de reorganización social' [más que las nuevas tecnologías de comunicación] es un nuevo modelo económico y político con intenciones globalizadoras y transnacionales" y que "el surgimiento de nuevos actores sociales asociados a prácticas socioculturales nuevas, es un área de investigación que vale la pena abordar".

La afirmación 12, que propone un principio estratégico para la difusión social de los productos de la investigación alcanza un grado muy alto, de acuerdo, pero despierta más escepticismo que entusiasmo entre los sujetos: "no siempre es posible, pero éste es un ideal a alcanzar". Por su parte, en la afirmación 33 se sugiere un incremento en la competencia académica como condición para "una mayor presencia e influencia político-práctica". El grado de acuerdo alcanza una media de 1.128 , y resalta la razón aducida por quienes están en desacuerdo: "la competencia teórico-metodológica es necesaria, pero no suficiente"; "es también un problema de poder"; "creo que va mucho más allá: la presencia política requiere también consolidaciones colectivas de agentes". La legitimación social, en suma, parece a los sujetos una meta clara pero lejana.

Por último, en relación con los procesos de asimilación/acomodación (capacidad de ajuste/aprendizaje) del campo académico en su entorno social, se agruparon tres afirmaciones $\left(02,29\right.$ y 34) ${ }^{24}$ La primera ennumera criterios

los potenciales usuarios de esos conocimientos y el público en general" (Raúl Fuentes y Enrique Sánchez, 1989). (33) "Solamente ganando un cada vez mayor respeto en el campo de nuestra competencia - el uso de herramientas teóricas, metodológicas, etc. para la producción de una cada vez mayor información sobre la comunicación social, ganaremos cada vez una mayor presencia e influencia político-práctica" (Enrique Sánchez Ruiz, 1987).

24. (02) "Es necesario evaluar nuestra práctica social con estándares y criterios como el rigor metodológico de nuestros planteamientos, la consistencia lógica de nuestros argumentos, la 
científicos generales de evaluación de las prácticas de investigación, y alcanza un

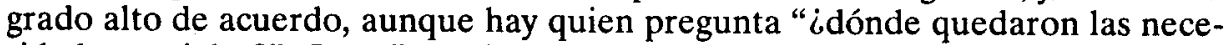
sidades sociales?". La afirmación 34 define, a escala latinoamericana, como "preocupación principal, que parece superar a la curiosidad científica", a la necesidad de "intervención sobre la realidad" de los investigadores de la comunicación. El índice de acuerdo es medio, pues "las excepciones tienden a multiplicarse"; "varía según las líneas y corrientes de investigación" y porque resulta una "afirmación válida para los setenta y parte de los ochenta nomás".

Para algunos de los sujetos "se viene atenuando la polarización de las posturas en función de conocer y transformar una realidad multidimensional y compleja"; en tanto que "el investigador no puede abstraerse de sus propias condiciones de producción. El reto es cómo equilibrar las tomas de posición con el rigor teórico-metodológico", pues "ambas posiciones no son excluyentes, se les ha tendido a desvincular de manera artificiosa" y "ésa es la meta de la investigación en ciencias sociales" "preocuparse en responder a los requerimientos político-sociales".

Finalmente, la afirmación 29 confronta directamente la utilidad y pertinencia de la investigación académica de la comunicación en México, asegurando que "no pesa", debido a que no hay "vínculos de diseminación" y a su mala calidad. El grado de acuerdo de los investigadores académicos es apenas medio, pues hay reacciones de distintos tipos: algunas de aceptación de tal diagnóstico “en líneas generales. Obviamente hay excepciones"; o "además de que hay poca consolidación de posiciones y protagonismos colectivos que permitan contar con una presencia relevante en los diversos espacios e instituciones sociales"; otros comentarios aportan matices: "hay, sin embargo, algunos casos de gran calidad. Creo que el problema básico es la falta de recursos, además de la gran dispersión de los investigadores y el celo absurdo sobre su trabajo y, por último, la mínima difusión de las investigaciones". Hay también dos argumentos en la base de los desacuerdos: por un lado, la afirmación es "muy radical. La investigación pesa en varios campos"; por otro, "si yo creyera esto no estaría haciendo investigación. El reto es lograr que nuestra incidencia sea mayor cada día".

honestidad intelectual de nuestras investigaciones y la base empírica de nuestros estudios" (Abraham Nosnik, 1988). (29) "La investigación académica no pesa ni siquiera en la academia, mucho menos en las empresas o en el gobierno. En parte porque no hay vínculos de diseminación de la información y en parte porque la calidad de la investigación deja mucho que desear" (Rubén Jara, 1988). (34) "El investigador de la comunicación y la cultura latinoamericanas, a diferencia de sus pares europeos o norteamericanos, trabaja a partir de una toma de posición, y su producción refleja los momentos de la coyuntura política. En ningún momento, ni siquiera en los periodos de mayor ingenuidad, este intelectual parece haber trabajado y producido sin preocuparse en responder a los requerimientos político-sociales. La preocupación principal, que parece superar a la curiosidad científica, es la necesidad de intervención en la realidad. Las excepciones parecen confirmar la regla" (Luis Gonzaga Motta, 1989). 


\section{Indicadores de la reflexividad}

Esta extensa exploración analítica de las representaciones discursivas (tanto en producción como en reconocimiento) de algunos aspectos del campo académico y de la práctica de la investigación de la comunicación en México, expresadas por un grupo considerable de investigadores, permite identificar algunos de los rasgos principales de un perfil ideológico o formación discursiva específicamente representativos de ese grupo, tanto en lo que tienden a compartir como en los ejes de divergencia de sentido, o de pugna por la hegemonía, operantes. Estos rasgos indican también, de acuerdo con el marco metodológico adoptado, los ingredientes cognoscitivos que, a falta de consensos sólidos en lo teóricometodológico, constituyen las bases de una identidad profesional (más o menos) compartida por los sujetos, en tanto investigadores de la comunicación, especialmente bajo la forma de normas éticas y de estilos de pensamiento propios del campo.

Para el conjunto de los investigadores encuestados parecen ser claras las condiciones generales que obstaculizan o limitan sus prácticas de investigación. Los tres géneros de condiciones postulados (Fuentes, 1995) como "contexto triple de la estructuración" del campo académico, especialmente como determinaciones socioculturales externamente impuestas a esta estructuración (la inconsistencia disciplinaria, la dependencia estructural y la crisis universitaria), son mayoritariamente asumidos y reconocidos por los investigadores, aunque las interpretaciones sobre sus causas y sobre lo que tendría que hacerse ante ellas difieren (en algunos aspectos hasta alcanzar una auténtica polarización de posturas).

En los comentarios a las afirmaciones se insinúa una tensión fuerte entre la identificación institucional y las representaciones negativas sobre la articulación interinstitucional del campo. Son sobre todo muy elocuentes el rechazo a la imagen de la comunidad académica latinoamericana y el descrédito de la AMIC. Todo ello refuerza la impresión de una tendencia hacia el individualismo, más que a un sentido de comunidad, sin embargo también presente con cierta fuerza. La coincidencia en la identificación de ciertos rasgos y en el reconocimiento de situaciones como el cambio tecnológico-cultural (globalización) de las comunicaciones, la transformación político-económica (neoliberal) del entorno, la insuficiencia de los recursos para la investigación académica y la propia "debilidad" disciplinaria del campo, coexisten entre los investigadores, con dudas o posiciones polarizadas en cuanto a la orientación y justificación social del trabajo de investigación, las fundamentaciones teórico-metodológicas y, sobre todo, con respecto a la organización concreta del campo y la viabilidad de los objetivos colectivos anteriormente sostenidos.

La continuidad utópica, sin ser abandonada, parece ser puesta en cuestión por una buena parte de los sujetos (al menos en referencia a algunos aspectos), y la autonomía intelectual, postulada como meta colectiva, ahora parece aplicarse mucho más a escala individual. No puede ignorarse que la crisis es una de las 
constantes más fuertes en el discurso (y en la experiencia) de los sujetos, por lo que los "procesos de asimilación/acomodación del sentido (utópico) del campo y las prácticas en el cambiante entorno sociocultural de la "realidad" no pueden desarticularse de los "procesos de constitución de los sujetos" y de los de "formación/conformación del habitus" que les es propio, en la explicación de los procesos (intermedios) de institucionalización y profesionalización.

Como se ha señalado, en el estudio de donde se extrae este trabajo se trató de analizar la determinación social del sentido de prácticas situadas estructuralmente, en un proceso multidimensional que al mismo tiempo que ha impulsado la estructuración del campo académico de la investigación de la comunicación en México, ha definido los rasgos distintivos de la constitución de los sujetos como agentes en el campo (Fuentes, 1995). Los elementos más ricos para elaborar la reconstrucción de la configuración cognoscitiva de este campo, provienen de 24 entrevistas abiertas (véase Anexo) en que se buscó sintetizar las representaciones de los investigadores en tres dimensiones interpretativas: sobre las trayectorias profesionales personales, las identidades o habitus profesionales, y la proyección utópica del trabajo académico. En seguida se resumen algunos de los resultados del análisis de este último aspecto.

El juicio colectivo sobre las condiciones de origen del campo académico es en buena medida homogéneo, tanto en lo que respecta a la reconstrucción de la inserción personal en él, como en cuanto a una caracterización de la época y lugar. No deja de percibirse una mezcla de nostalgia y desencanto, en muchos casos explícitamente formuladas y asumidas como tales, en el discurso de los entrevistados acerca de esos años (los setenta), en que se ubican los orígenes del campo y de las trayectorias personales de los de mayor edad. Ésa fue, también, una época de conflictos, que veinte años después son más fríamente analizados por sus protagonistas.

La lucha por "el monopolio del saber legítimo" (Bourdieu, 1988a) en el origen del campo de la investigación académica de la comunicación en México, fue entablada sobre todo entre quienes buscaban enraizar los modelos "científicos" empiristas y quienes radicalizaron el compromiso académico con la transformación social, según el modelo de la militancia latinoamericana. Esta época de conflictos (que se sitúa entre 1976 y 1982) marcó sin duda los límites sobre los que habría de seguirse desarrollando el campo, pero también contribuyó determinantemente a configurar el habitus de los investigadores, y a generar entre los protagonistas más directos al mismo tiempo enormes aprendizajes y distancias personales casi insalvables. Sin embargo, puede decirse que los sujetos comparten la sensación de que en esta lucha todos resultaron perdedores, ya que su reconstrucción de "la crisis de los ochenta", matices aparte, es muy homogénea, sobre todo en cuanto a la investigación como profesión.

El diagnóstico colectivo sobre las condiciones para el desarrollo académico en los años ochenta, especialmente formulado en términos institucionales (infraestructuras y políticas universitarias) y laborales (remuneración), concluye en reconstrucciones más alentadoras de la "situación actual". La implicación de analizar explícitamente las razones por las cuales la mayor parte de los investi- 
gadores buscó ingresos complementarios, pero no dejó la universidad, permite explicar, mediante la abducción de algunos rasgos propios del habitus específico, tanto esta permanencia (de hecho) como la lógica de la oposición entre satisfacción personal e insatisfacción económica, o los condicionantes de los rechazos individuales a asumir el liderazgo en el campo, proyectados en otros instrumentos del mismo estudio. En el contexto en que fueron formuladas estas reconstrucciones, los investigadores enfatizaron la fuerza de la satisfacción personal y una manera de asumir el compromiso con la profesión como todo un proyecto de vida.

Si en los años setenta, entonces, se conjuntó una serie de factores contextuales (económicos, políticos, culturales) que facilitaron la emergencia y el desarrollo de una "utopía comunicacional" (profesional y social) a la cual se adscribieron muchos de quienes optaron por la carrera académica, y por ello en buena medida la investigación como proyecto se convirtió, de entrada, en un espacio de conflicto, en los años ochenta el cambio en esos factores contextuales (crisis nacional), que además coincidió con el crecimiento desmedido de los programas de licenciatura y con la generalización de la conciencia de la "debilidad" tanto científica como política de la investigación de la comunicación, determinó una reconfiguración del campo académico como estructura institucionalizada, que en lo cognoscitivo implicó a su vez un ajuste en el habitus comunitario y un mayor grado de exigencia interna, aunque eso signifique una disminución del número de investigadores. De ahí sale la representación tanto del "estado actual" del campo, como de su futuro previsible, donde vuelve a presentarse un alto grado de consenso.

Creo que vamos a estar muy pocos haciendo investigación académica en el futuro, pero los pocos vamos a estar con mejores posibilidades de hacerla. Y a lo mejor va a ser una investigación más importante, en términos de que va a ser una investigación mucho más precisa, mucho más vinculada, menos allá en la estratósfera y que pueda aportar más cosas, tener una incidencia mayor aunque seamos menos [entrevista de investigación].

La preocupación por la dispersión y por la debilidad del campo de la investigación se encuentra muy generalizada, y en las entrevistas es asociada con tres tipos de factores: por un lado, los institucionales (falta de apoyo al campo); por otro, los teórico-metodológicos (indefinición del objeto); y también, con factores interpersonales (aislamiento, conflictos). Con soprendente frecuencia, los sujetos más antiguos en el campo hablaron de "reconciliación" como deseo personal compartido, pero también de la prevalencia de la "envidia" como actitud generalizada, a la cual asociaron - casi todos - directamente tanto la debilidad del campo como el aislamiento individual percibidos.

Es muy notable la general consideración de que el trabajo de investigación es "solitario y aislado", tanto en razón de las condiciones "objetivas" como sobre todo por causas intersubjetivas (envidias, conflictos), aunque también "por preferencias personales". Por ello puede interpretarse que el aislamiento objetivo 
es bastante menor que el percibido por los sujetos. A lo largo de más de un año en que se realizaron las entrevistas, el entrevistador fue sorprendiéndose cada vez más de la homogeneidad de las representaciones al respecto, que cada uno de los investigadores caracterizaba como "una impresión muy personal". Éste y otros rasgos son compartidos por la mayoría de los entrevistados, como parte de su habitus específico, sin que los sujetos sean conscientes de ello.

Por otro lado, la preocupación de los investigadores "establecidos" por la reproducción del campo es compartida, casi en los mismos términos, por los pocos, más jóvenes, que se habían incorporado en los últimos años al campo (en 1993, cuando se realizaron las entrevistas), y en general, éste es percibido de una manera poco autocomplaciente por la mayoría de los investigadores entrevistados; en este rasgo de las representaciones hay también una amplia coincidencia, independientemente de la antigüedad de los sujetos.

La identidad profesional de los investigadores de la comunicación como campo académico, está sujeta aún, según ellos mismos, a un proceso de construcción para el cual parecen estar sentadas las bases y en función del cual es necesario instrumentalizar el conocimiento producido en dos sentidos opuestos pero complementarios: hacia "afuera", en términos de responsabilidad social, como estrategia de legitimación (política), y hacia "adentro", en términos metodológicos, como condición para la profesionalización (científica). En la articulación entre uno y otro sentidos, la ideología profesional, el habitus, la "matriz disciplinaria" operante como "núcleo común de sentido básico compartido" en las prácticas estructuradoras de los sujetos, revelan una ética claramente definida y profundamente enraizada, aunque insuficientemente formulada, quizá por ser la lógica subyacente más profunda de articulación del sentido.

Pero la lucha por el prestigio (individual y colectivo) es sin duda un factor clave para entender los procesos multidimensionales de estructuración del campo de la investigación académica de la comunicación en México. Este factor, manifiesto en innumerables estrategias conductuales y discursivas (políticas tanto como científicas) de los sujetos, ha desembocado en una tensión fuertemente equilibrada (y que por lo tanto tiende a la inmovilidad) entre "la competencia por logros creativos" y "la competencia por la monopolización del saber legítimo" (Knorr-Cetina, 1981: 70) entre muy pocos agentes (personales e institucionales) que han internalizado las condiciones objetivas externamente impuestas al campo y a sus prácticas (la "inconsistencia disciplinaria", la "dependencia estructural" y la "crisis universitaria") y han mantenido como instrumentos (recursos y esquemas interpretativos) de su agencia estructuradora (Giddens, 1984) los proyectos fundacionales de la institucionalización académica, la autonomía intelectual y la continuidad utópica hasta un momento que parece ser (así es percibido por los sujetos) un límite histórico de viabilidad. Por ello, sin que los sujetos sean conscientes de ello, nadie asume subjetivamente (pudiéndolo objetivamente hacer) el papel de liderazgo intelectual y político que por la vía del prestigio pueda generar poder legítimo (Sewell, 1992) tanto al "interior" como al "exterior", como exige el campo para sobrevivir como tal. 
La interpretación, prácticamente unánime entre los sujetos entrevistados, sobre la debilidad disciplinaria del campo, y al mismo tiempo, sobre la relevancia creciente en términos socioculturales del objeto de estudio, apunta hacia una reinterpretación más profunda de los factores de la (re)configuración cognoscitiva del campo, en una dimensión ética, que la sociología de la ciencia ha explorado menos y que, como señala A. Pickering (1992), implica el paso definitivo de una concepción de la ciencia como conocimiento a una concepción de la actividad científica como práctica socioculturalmente determinada y éticamente orientada, donde los sujetos generan y regeneran continuamente el sentido. A partir de este eje, el estudio de donde se extrae esta presentación concluye con la propuesta de un modelo de la estructuración/desestructuración/reestructuración del campo, en que se enfatizan las opciones vigentes para su legitimación académica y social, según se pueden situar objetivamente y según se las representan los sujetos que son sus agentes (este modelo puede verse también en Fuentes, 1997).

\section{Bibliografía}

Bourdieu, Pierre (1988a). Homo academicus. Stanford University Press, California.

- (1988b). Cosas dichas, Gedisa, Buenos Aires.

Clark, Burton R. (1992). El sistema de educación superior. Una visión comparativa de la organización académica, Nueva Imagen/Universidad Futura/UAMAzcapotzalco, México.

Fleck, Ludvik (1979). Genesis and development of a scientific fact, Thaddeus J. Trenn y Robert K. Merton (eds.), The University of Chicago Press, Chicago y Londres.

Fuentes Navarro, Raúl (1995). "La emergencia de un campo académico: continuidad utópica y estructuráción científica de la investigación de la comunicación en México", tesis de doctorado en ciencias sociales, Universidad de Guadalajara, Guadalajara.

(1996a). La investigación de la comunicación en México. Sistematización documental 1986-1994, ITESO/Universidad de Guadalajara, Guadalajara.

(1996b). "Un acercamiento bibliométrico a la configuración cognoscitiva del campo académico de la comunicación en México", en Comunicación y Sociedad, núm. 27, DECs-Universidad de Guadalajara, pp. 11-41.

(1997). "Consolidación y fragmentación de la investigación de la comunicación en México, 1987-1997", en Comunicación y Sociedad, núm. 30, DECSUniversidad de Guadalajara, pp. 27-50.

Giddens, Anthony (1984). The constitution of society. Outline of the theory of structuration, University of California Press, Berkeley y Los Angeles. 
Knorr-Cetina, Karin (1981). The manufacture of knowledge. An essay on the constructivist and contextual nature of science, Pergamon Press, Oxford.

Kuhn, Thomas S. (1982). La tensión esencial. Estudios selectos sobre la tradición y el cambio en el ámbito de la ciencia, Fondo de Cultura Económica/conACyT, México.

Masterman, Margaret (1970). “The nature of a paradigm”, en Lakatos y Musgrave (eds.), Criticism and the growth of knowledge, Cambridge University Press, Cambridge.

Pickering, Andrew (1992). "From science as knowledge to science as practice", en Pickering (ed.), Science as practice and culture, The University of Chicago Press, Chicago y Londres.

Sewell, Jr. William H. (1992). "A theory of structure. Duality, agency and transformation", en American Journal of Sociology, vol. 98, núm. 1, pp. 1-29.

Verón, Eliseo (1987). La semiosis social. Fragmentos de una teoría de la discursividad, Gedisa, Buenos Aires.

\section{Anexo}

Investigadores encuestados/entrevistados

Francisco de Jesús Aceves González

Áurea Blanca Aguilar Plata

Eduardo Andión Gamboa

Mauricio Andión Gamboa

Rosa María Aponte Herrera

Pablo Arredondo Ramírez

Consuelo Beas Oropeza

Claudia Benassini Félix

Pablo Casares Arrangoiz

María de la Luz Casas Pérez

Rafael Castro y Lluriá

Mercedes Charles Creel

Inés Cornejo Portugal

Carlos Corrales Díaz

Delia María Crovi Druetta

Adrián de Garay Sánchez

María Josefa Erreguerena Albateiro

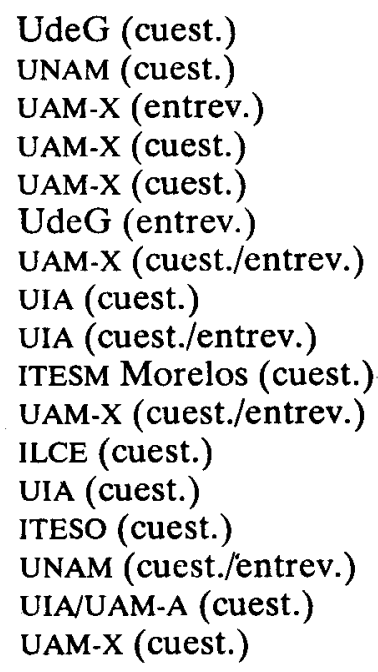

UdeG (cuest.)

UNAM (cuest.)

UAM-X (entrev.)

UAM-X (cuest.)

UAM-X (cuest.)

UdeG (entrev.)

UAM-X (cuest./entrev.)

UIA (cuest.)

UIA (cuest./entrev.)

ITESM Morelos (cuest.)

UAM-X (cuest./entrev.)

ILCE (cuest.)

UIA (cuest.)

ITESO (cuest.)

UNAM (cuest./entrev.)

UIA/UAM-A (cuest.)

UAM-X (cuest.) 
Javier Esteinou Madrid

Fátima Fernández Christlieb

Gilberto Fregoso Peralta

Raúl Fuentes Navarro

L. Jesús Galindo Cáceres

Carola I. García Calderón

Carlos Gómez-Palacio Campos

Jorge A. González Sánchez

Enrique Guinsberg Blank José Rubén Jara Elías José Carlos Lozano Rendón Carlos E. Luna Cortés

Carlos Maya Obé

Guillermo Orozco Gómez

C. Patricia Ortega Ramírez

José Antonio Paoli Bolio

Romeo Pardo Pacheco

Francisco Prieto Echaso

Rossana Reguillo Cruz

Martha Renero Quintanar

Soledad Robina Bustos

Cecilia Rodríguez Dorantes

Cristina Romo de Rosell

Josep Rota Burgueño

Enrique E. Sánchez Ruiz

Beatriz A. Solís Leree

Florence Toussaint Alcaraz

Teresa de Jesús Tovar Peña

Raúl Trejo Delarbre

Josefina Vilar Alcalde

Margarita Yépez Hernández

Lauro J. Zavala Alvarado

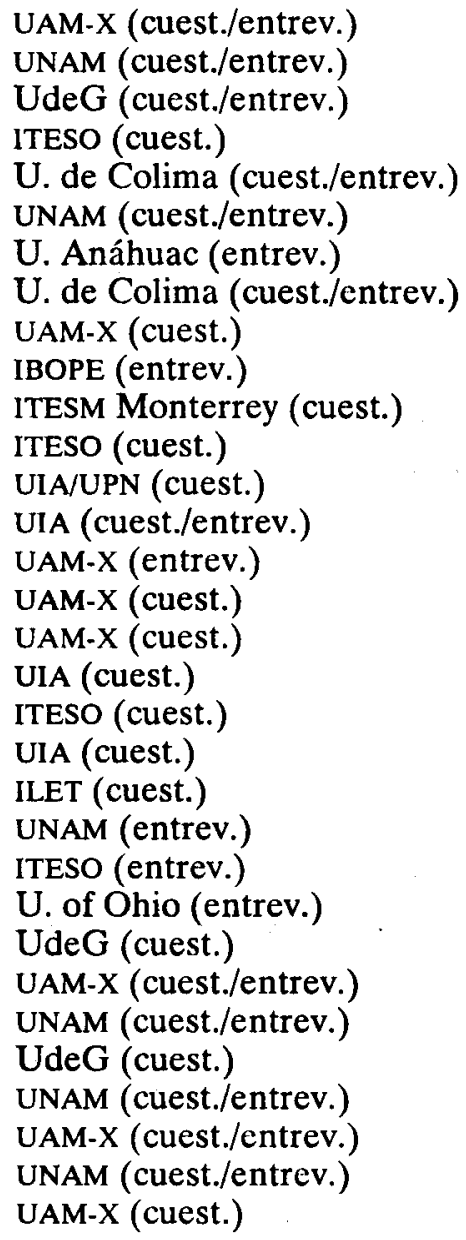

